\title{
Refractive correction method for digital charge-coupled device-recorded Scheimpflug photographs by means of ray tracing
}

\author{
Wolfgang Fink \\ University of Southern California \\ Doheny Eye Institute at the Keck School of Medicine \\ Los Angeles, California 90033 \\ and \\ California Institute of Technology \\ Pasadena, California 91125 \\ E-mail:wfink@autonomy.caltech.edu
}

\begin{abstract}
Our purpose is to correct digital CCD-recorded Scheimpflug photographs, imaging both the anterior and posterior corneal surface, the anterior chamber, and the anterior eye lens surface for optical distortions. In a ray-tracing algorithm the imaging of the posterior corneal surface in a given Scheimpflug photograph is corrected by applying Snell's law on parallel incident rays entering through the anterior corneal surface. Once the posterior corneal surface is corrected, the procedure is repeated, again with parallel incident rays entering through both the anterior and now corrected posterior corneal surface, to correct the imaging of the anterior eye lens surface. The refractive indices necessary for Snell's law are taken from Gullstrand's exact schematic eye model. Due to the optical/refractive correction, the digital Scheimpflug photograph decreases in size perpendicular to the direction of the optical axis. As a consequence the curvature radii of both the posterior corneal surface and the anterior lens surface are reduced significantly, as compared to the original digital Scheimpflug photograph. Furthermore, the corneal thickness and the anterior chamber depth are increased. The presented refractive correction method enables us to extract from Scheimpflug photographs the following quantities rather realistically: structure coordinates and curvature radii of both the posterior corneal surface and the anterior lens surface, corneal thickness, and anterior chamber depth. This method can readily be applied to other imaged quantities, such as the posterior eye lens surface, the lens thickness, and the pupillary opening. (c) 2005 Society of Photo-Optical Instrumentation Engineers. [DOI: $10.1117 / 1.1899683]$
\end{abstract}

Keywords: accommodation; corneal topography; image analysis; refractive error; refractive surgery; Scheimpflug photography; refractive correction.

Paper 03113 received Sep. 16, 2003; revised manuscript received Feb. 18, 2004; accepted for publication Aug. 9, 2004; published online Apr. 13, 2005.

\section{Introduction}

Images taken by a camera based on the Scheimpflug principle $^{1}$ (see Fig. 1 and Fig. 2 in Sec. 2) can already provide valuable information without considering distortions. However, for a quantitative evaluation, for which especially digital Scheimpflug photographs are suitable, optical/refractive distortions must be taken into account. Such distortions arise not only from the anterior corneal surface (ACS) alone; images of deeper structures of the eye such as the posterior corneal surface, the pupil, and the anterior lens surface are also distorted $^{2-5}$ due to the differences in refractive indices and the various radii of curvature of the refractive surfaces.

Quantitative information that can be retrieved after performing optical/refractive corrections includes realistic estimates of the following biometric quantities within the eye:

Address all correspondence to Wolfgang Fink, University of Southern California, Doheny Eye Institute at the Keck School of Medicine, Los Angeles, California 90033, California Institute of Technology, Pasadena, California 91125. E-mail: wfink@autonomy.caltech.edu
1. structure coordinates of the posterior corneal surface

2. curvature radius of the posterior corneal surface (see also Dubbelman et al. ${ }^{6}$ )

3. corneal thickness

4. pupillary diameter

5. structure coordinates of the anterior lens surface

6. curvature radius of the anterior lens surface (see also Dubbelman and van der Heijde ${ }^{7}$ )

7. anterior chamber depth

and, if the quality and structural depth of the Scheimpflug image permits,

1. structure coordinates of the posterior lens surface

2. curvature radius of the posterior lens surface (see also Dubbelman and van der Heijde ${ }^{7}$ )

1083-3668/2005/\$22.00 @ 2005 SPIE 


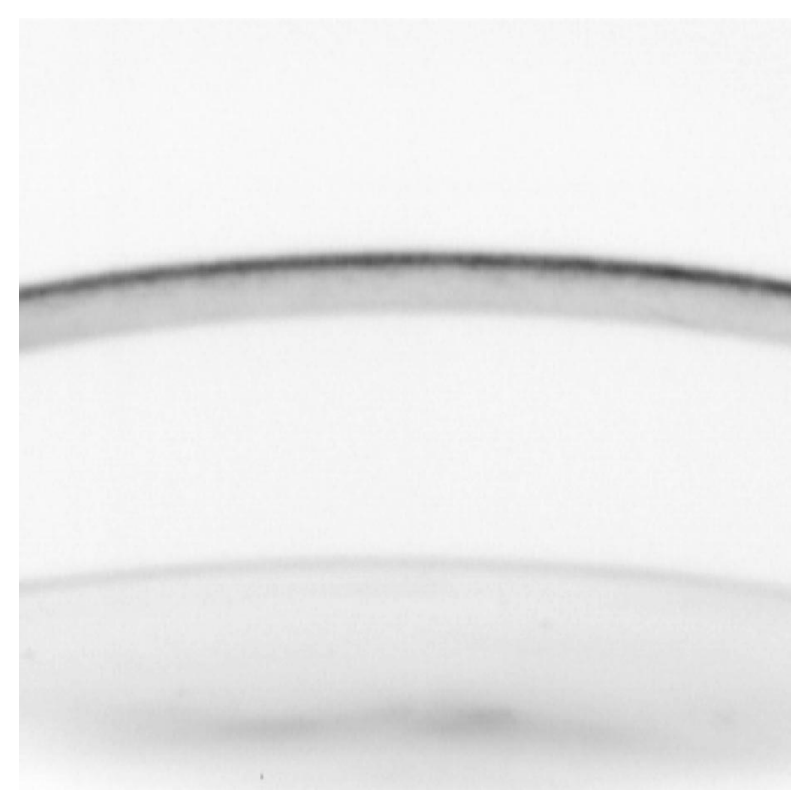

Fig. 1 Digital-CCD-recorded Scheimpflug photograph (512×512 pixels, inverted gray scale for enhanced picture quality, taken from Fink et al. ${ }^{2}$.

\section{3. thickness of the lens (see also Dubbelman et al. ${ }^{8}$ )}

Of special interest among those quantities is the estimation of the curvature radius of the anterior lens surface since it is possible now to monitor its change under various stages of accommodation, thus exhibiting the changes in shape and location of the crystalline lens (see also Dubbelman et al. ${ }^{9}$ ).

In earlier work, e.g., by Richards et al. ${ }^{4}$ and Kampfer et al., ${ }^{5}$ optical corrections have been applied to scanned Scheimpflug photographs. In their work, the authors had to overcome problems such as photo/camera distortion and distortions resulting from projection of Scheimpflug negatives for digitization in addition to the natural refractive distortion of the eye.

Progress has now been made on the experimental side by providing digital-CCD-recorded Scheimpflug photographs ${ }^{2,3,10}$ (see Fig. 1), thus eliminating the additional problems already mentioned, of course, with the exception of the natural refractive distortion of the eye. ${ }^{*}$ An algorithm developed by Huebscher et al., ${ }^{3}$ Huebscher and Schmidt, ${ }^{10}$ and Hachicha et al. ${ }^{11}$ determines the seeming structure coordinates of the various refractive surfaces in the digital Scheimpflug record by means of a gray-scale-threshold-filter method, taking the camera distortion into account. In this paper, a raytracing algorithm is presented ${ }^{2,12-15}$ that starts from this preprocessed data file and performs the optical/refractive

*Reviewer A's annotation: "The now-unavailable Zeiss Scheimpflug SL-C System (using a video camera wherein the image was digitized), modified by several users (notably the teams headed by Prof. Dr. Basil Worgul of Columbia University and Dr. Manuel Datiles of the National Eye Institute of the U.S. National Institutes of Health) and originally manufactured in Oberkochen, Germany, featured a unique calibration system. This system was based on an Ulbricht sphere attachment for both optical and geometric calibration. Using this Ulbricht sphere, one can overcome most of the refractive and optical deficiencies of the Scheimpflug system."

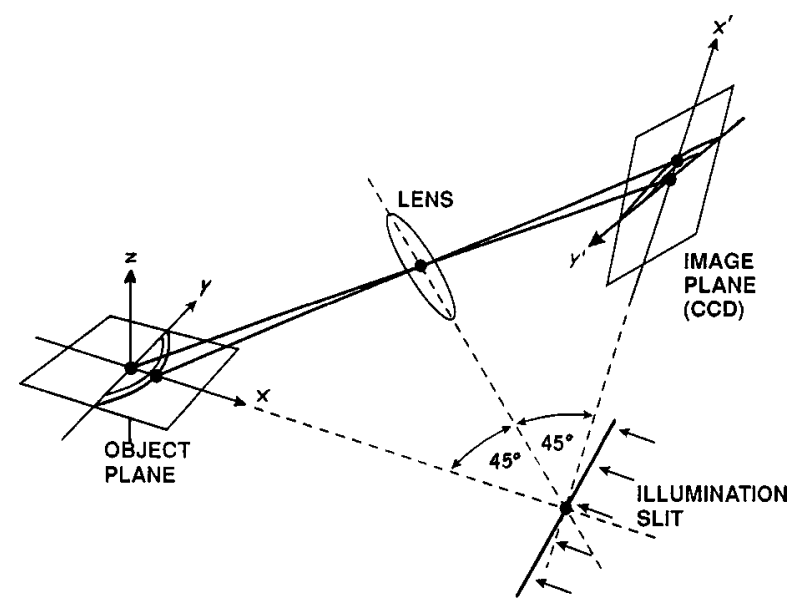

Fig. 2 Principle of Scheimpflug imaging (after Richards et al. ${ }^{4}$ ).

corrections to obtain the "true" locations of both the posterior corneal surface denoted as PCS and the anterior lens surface denoted as ALS.

This investigation was initiated by discussions with Huebscher and Seiler. Numerical results were presented in part in Fink et al. ${ }^{2}$ The underlying method/algorithm, which is presented in detail in this paper, is tested with the same experimental data that were already used in Fink et al. ${ }^{2}$

\section{Methods}

To receive more realistic coordinates of the posterior corneal surface, the refraction that takes place at the anterior corneal surface must be taken into account. Therefore, the seeming structure coordinates of the posterior corneal surface, which are derived from the original digital Scheimpflug record via the already mentioned gray-scale-threshold-filter method, ${ }^{3,10,11}$ must be corrected. In the following, this preprocessed data file will be simply referred to as the Scheimpflug record.

Since the Scheimpflug camera faces the eye under examination in an angle of $45 \mathrm{deg}$ with respect to the optical axis, the pathway of incident rays is always 3-D. Figure 2 illustrates the principle of Scheimpflug imaging. Figure 3 shows the proposed refractive correction procedure in a simplified 2-D depiction. Figures 2 and 4 schematically introduce some of the variables used in the following mathematical derivation of the refractive correction method.

We assume in the following that the ACS structure coordinates can be directly obtained from the Scheimpflug record without any further refractive correction since the imaging light rays for the ACS are directly reflected and not undergoing refraction, i.e., we ignore, for simplicity, the refraction taking place at the transition from the ACS to the tear film. First, according to Figs. 2 to 4, the intersection points of the parallel light rays, which image the PCS, with the ACS have to be calculated. Assuming that the borderline of the ACS is located in the $(z=0)$ plane, the equation for the image rays that form the image of the PCS in an angle of 45 deg to the $(x / y)$ plane, is given by 


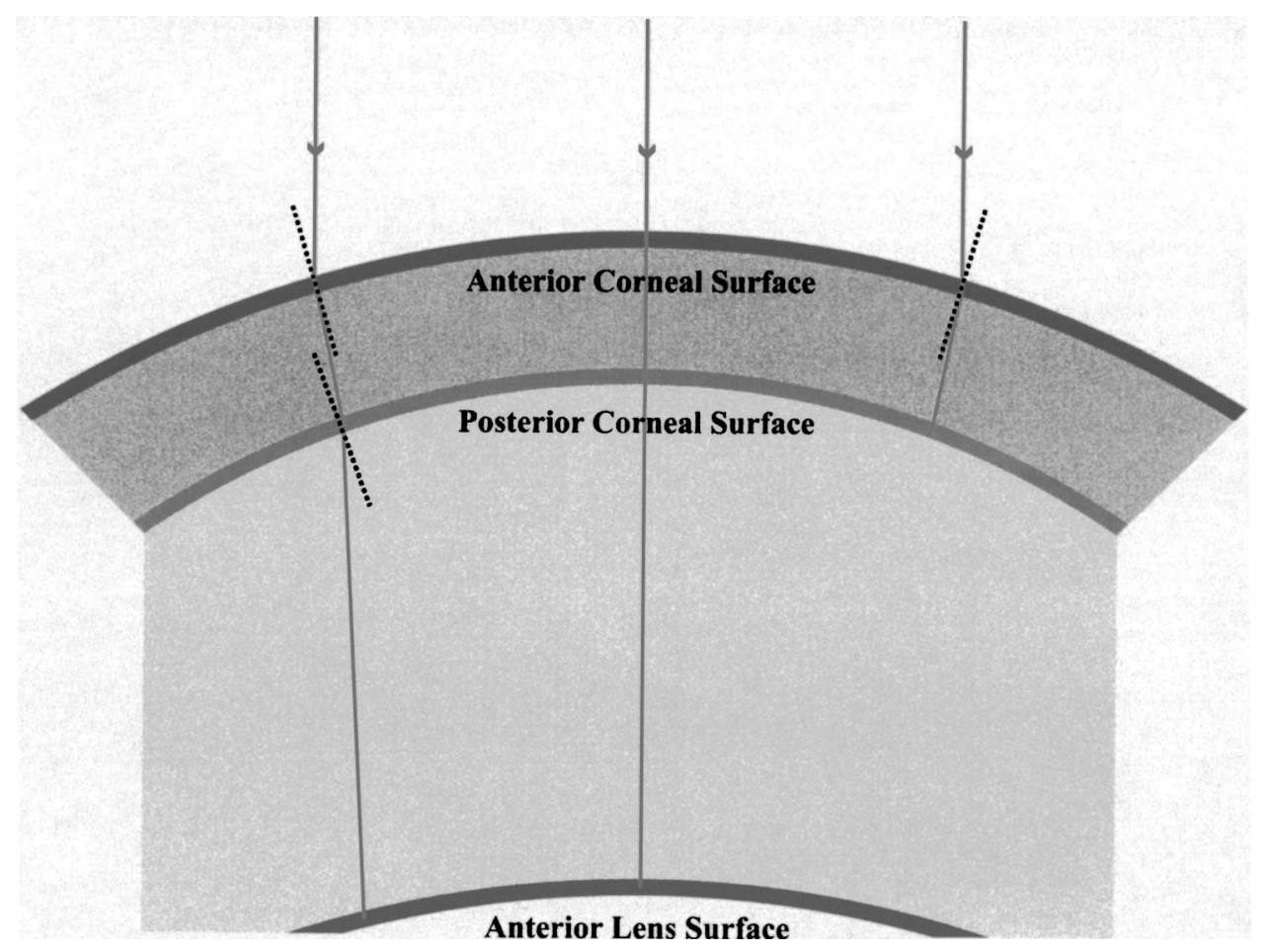

Fig. 3 Principle of refractive correction of the posterior corneal surface taking refraction at the anterior corneal surface into account and refractive correction of the anterior lens surface taking refraction at both the anterior corneal surface and the corrected posterior corneal surface into account.

$$
\mathbf{g}_{\mathrm{PCS}}(\mu)=\left(\begin{array}{c}
H \\
S \\
P
\end{array}\right)+\mu g_{\mathrm{PCS}}^{\dagger}
$$

where

$$
g_{\mathrm{PCS}}^{\dagger}=\frac{1}{\sqrt{2}}\left(\begin{array}{l}
1 \\
0 \\
1
\end{array}\right)
$$

is the unit vector in the $(x / z)$ plane which forms an angle of 45 deg with the $(x / y)$ plane (see Fig. 4$) ; S$ is the uncorrected distance of each parallel light ray from the optical axis in the Scheimpflug record; $P$ is the uncorrected distance between ACS and PCS in the same image row; and $H$ is given by the equation $H=\left(R_{\mathrm{ACS}}^{2}-S^{2}\right)^{1 / 2}$.

Considering only the central part of the cornea (up to 4 $\mathrm{mm}$ diameter), a spherical approximation is reasonable. ${ }^{16}$ Thus, any point of the anterior corneal surface is given by spherical coordinates

$$
\mathbf{C}_{\mathrm{ACS}}=\left(\begin{array}{l}
R_{\mathrm{ACS}} \cos \phi \sin \theta \\
R_{\mathrm{ACS}} \sin \phi \sin \theta \\
R_{\mathrm{ACS}} \cos \theta
\end{array}\right),
$$

where the curvature radius $R_{\mathrm{ACS}}$ of the anterior corneal surface is derived from a circle fit routine, ${ }^{17}$ which is applied to the surface data of the ACS in the Scheimpflug record. The intersection of the image ray $\mathbf{g}_{\mathrm{PCS}}$ with the ACS can now be found numerically by solving the equation

$$
\mathbf{g}_{\mathrm{PCS}}(\mu)=\mathbf{C}_{\mathrm{ACS}} \text {, }
$$

for $\mu$ by means of, e.g., a 3-D Newton-Raphson root-finding routine. ${ }^{18}$ Since there are two possible intersection points between a line and a sphere (apart from the tangential case) one must take the intersection point $\mathbf{g}_{\mathrm{PCS}}\left(\mu^{*}\right)$ that marks the entry to the ACS.

Going one step further, the surface normal to the ACS in that intersection point must be calculated. Defining the center

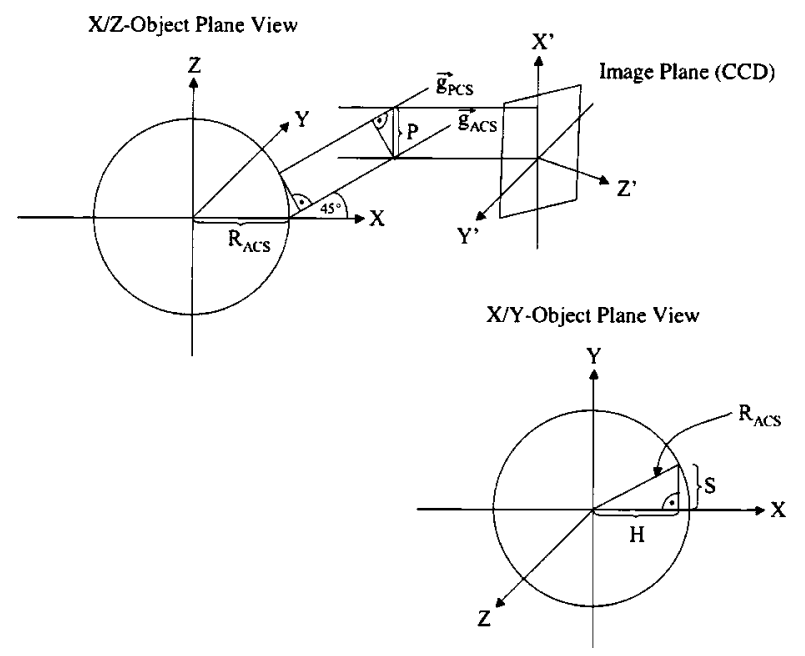

Fig. 4 Upper left: detailed $X / Z$ object plane view (see Fig. 2) with depiction of light rays for the anterior corneal surface (ACS) and uncorrected posterior corneal surface (PCS) originating from the (CCD) image plane; and lower right: detailed $X / Y$ object plane view (see Fig. 2) with further introduction of some of the variables used in the mathematical derivation of the refractive correction method. 
of the ACS sphere as the origin of the coordinate system the surface normal reads

$$
\mathbf{F}_{\perp \mathrm{ACS}}\left(\mu^{*}\right)=N \mathbf{g}_{\mathrm{PCS}}\left(\mu^{*}\right),
$$

with the normalization constant

$$
N=\frac{1}{\left[\left(H+\mu^{*} / \sqrt{2}\right)^{2}+S^{2}+\left(P+\mu^{*} / \sqrt{2}\right)^{2}\right]^{1 / 2}} .
$$

To apply Snell's law to the image ray $\vec{g}_{P C S}$, the angle $\alpha$ between the image ray and the surface normal $\mathbf{F}_{\perp \mathrm{ACS}}\left(\mu^{*}\right)$ before refraction has to be determined. A brief geometrical calculation yields

$$
\alpha=\arccos \left[\mathbf{F}_{\perp \mathrm{ACS}}\left(\mu^{*}\right) \frac{1}{\sqrt{2}}\left(\begin{array}{l}
1 \\
0 \\
1
\end{array}\right)\right] .
$$

With Snell's law the resulting angle $\beta$ after refraction is

$$
\beta=\arcsin \left(\frac{\sin (\alpha)}{n_{\text {cornea }}}\right),
$$

where $n_{\text {cornea }}$ denotes the refraction index within the cornea that is assumed to be constant in our model. The refraction index for the ambient air is set to 1 .

To determine the new direction vector $\mathbf{g}_{\mathrm{PCS}}^{\dagger \text { †new }}$ for the image ray $\mathbf{g}_{\mathrm{PCS}}$ after refraction at the ACS three conditions must be taken into account:

$$
\begin{gathered}
\mathbf{F}_{\perp \mathrm{ACS}}\left(\mu^{*}\right) \mathbf{g}_{\mathrm{PCS}}^{\dagger \text { new }}=\cos (\beta), \\
\mathbf{g}_{\mathrm{PCS}}^{\dagger \text { new }}\left[\mathbf{F}_{\perp \mathrm{ACS}}\left(\mu^{*}\right) \times \mathbf{g}_{\mathrm{PCS}}^{\dagger}\right]=0, \\
\left(\mathbf{g}_{\mathrm{PCS}}^{\dagger \text { †nw }}\right)_{x}^{2}+\left(\mathbf{g}_{\mathrm{PCS}}^{\dagger \text { new }}\right)_{y}^{2}+\left(\mathbf{g}_{\mathrm{PCS}}^{\dagger \text { new }}\right)_{z}^{2}=1 .
\end{gathered}
$$

The first condition is a consequence of Snell's law. The second condition mathematically describes the fact that the direction vector before $\left(\mathbf{g}_{\mathrm{PCS}}^{\dagger}\right)$ and after $\left(\mathbf{g}_{\mathrm{PCS}}^{\dagger \text { new }}\right)$ refraction as well as the surface normal $\mathbf{F}_{\perp \mathrm{ACS}}\left(\mu^{*}\right)$ are located in the same plane. The third condition expresses the normalization of the new direction vector $\mathbf{g}_{\mathrm{PCS}}^{\dagger \text { new }}$.

The new direction vector $\mathbf{g}_{\mathrm{PCS}}^{\text {tnew }}$ can be numerically obtained by solving the system of three equations [Eqs. (1) to (3)] by means of, e.g., a 3-D Newton-Raphson root-finding routine. ${ }^{18}$ Thus, the equation for the image ray after refraction at the ACS is as follows:

$$
\mathbf{g}_{\mathrm{PCS}}^{\mathrm{new}}(\gamma)=\mathbf{g}_{\mathrm{PCS}}\left(\mu^{*}\right)+\gamma \mathbf{g}_{\mathrm{PCS}}^{\dagger \text { new }} .
$$

Finally, to determine the corrected structure coordinate for the PCS the intersection of the new image ray with the $(z=0)$ plane must be calculated. Therefore, it is sufficient to solve the equation

$$
\left(\mathbf{g}_{\mathrm{PCS}}^{\text {new }}\right)_{z}(\gamma)=0
$$

for $\gamma$. The straightforward solution

$$
\gamma^{*}=-\frac{\mathbf{g}_{\mathrm{PCS}}\left(\mu^{*}\right)_{z}}{\left(\mathbf{g}_{\mathrm{PCS}}^{\dagger \text { new }}\right)_{z}}
$$

gives the new corrected structure coordinate $\mathbf{g}_{\mathrm{PCS}}^{\mathrm{new}}\left(\gamma^{*}\right)$ for the PCS (see Fig. 5 in Sec. 3).

The procedure described so far must be applied to each one of the parallel light rays that image the PCS in the digital Scheimpflug record. As a result, we obtain a refractive correction of the PCS in the digital Scheimpflug record that enables realistic estimations of corneal thickness and curvature radius $R_{\mathrm{PCS}}$ of the posterior corneal surface (see Fig. 5 in Sec. 3). The latter can be derived from a circle fit routine, ${ }^{17}$ which is applied to the now corrected surface data of the PCS.

Having corrected the structure coordinates for the posterior corneal surface as a first step, the same procedure as already described is repeated as a second step with parallel light rays that image the anterior lens surface. Now two refractions occur; one at the ACS and the other at the PCS taking into account the shape and refractive power of the anterior corneal surface and the corrected posterior corneal surface (see Fig. 3).

The calculation of the refraction that takes place at the ACS is identical to the procedure already described. The result is a new image ray that intersects the corrected posterior corneal surface at some point.

After determination of the intersection point with the corrected posterior corneal surface, the new direction vector for the image ray that underwent refraction at the PCS must be calculated. Then the new corrected structure coordinate for the ALS can be obtained from the intersection of the new image ray with the $(z=0)$ plane in a manner similar to that described for the PCS.

Note that the refraction index $n_{\text {anterior chamber }}=1.336$ currently used with Snell's law is assumed to be constant within the entire anterior chamber. Also assumed to be constant is the refraction index $n_{\text {cornea }}=1.376$ of the cornea. Both are taken from Gullstrand's exact schematic eye mode ${ }^{19}$ but can easily be adjusted to experimental data, when available.

We interpolate the posterior corneal surface data by a mathematical function and choose for simplicity a spherical approximation; since we are dealing with a correction, small deviations from the spherical approximation would influence the final results only in second order. Thus, any point of the PCS is given by the following spherical coordinates:

$$
\mathbf{C}_{\mathrm{PCS}}=\left(\begin{array}{l}
R_{\mathrm{PCS}} \cos \phi \sin \theta+C x_{\mathrm{PCS}} \\
R_{\mathrm{PCS}} \sin \phi \sin \theta+C y_{\mathrm{PCS}} \\
R_{\mathrm{PCS}} \cos \theta+C z_{\mathrm{PCS}}
\end{array}\right),
$$

where the curvature radius $R_{\mathrm{PCS}}$ of the posterior corneal surface as well as the center of the sphere

$$
\left(\begin{array}{c}
C x_{\mathrm{PCS}} \\
C y_{\mathrm{PCS}} \\
C z_{\mathrm{PCS}}
\end{array}\right)
$$

are derived from a circle fit routine, ${ }^{17}$ which is applied to the corrected surface data of the PCS.

Note that two digital Scheimpflug photographs in perpendicular planes of the same eye are required to determine the 3-D location of the center of the sphere that approximates the 


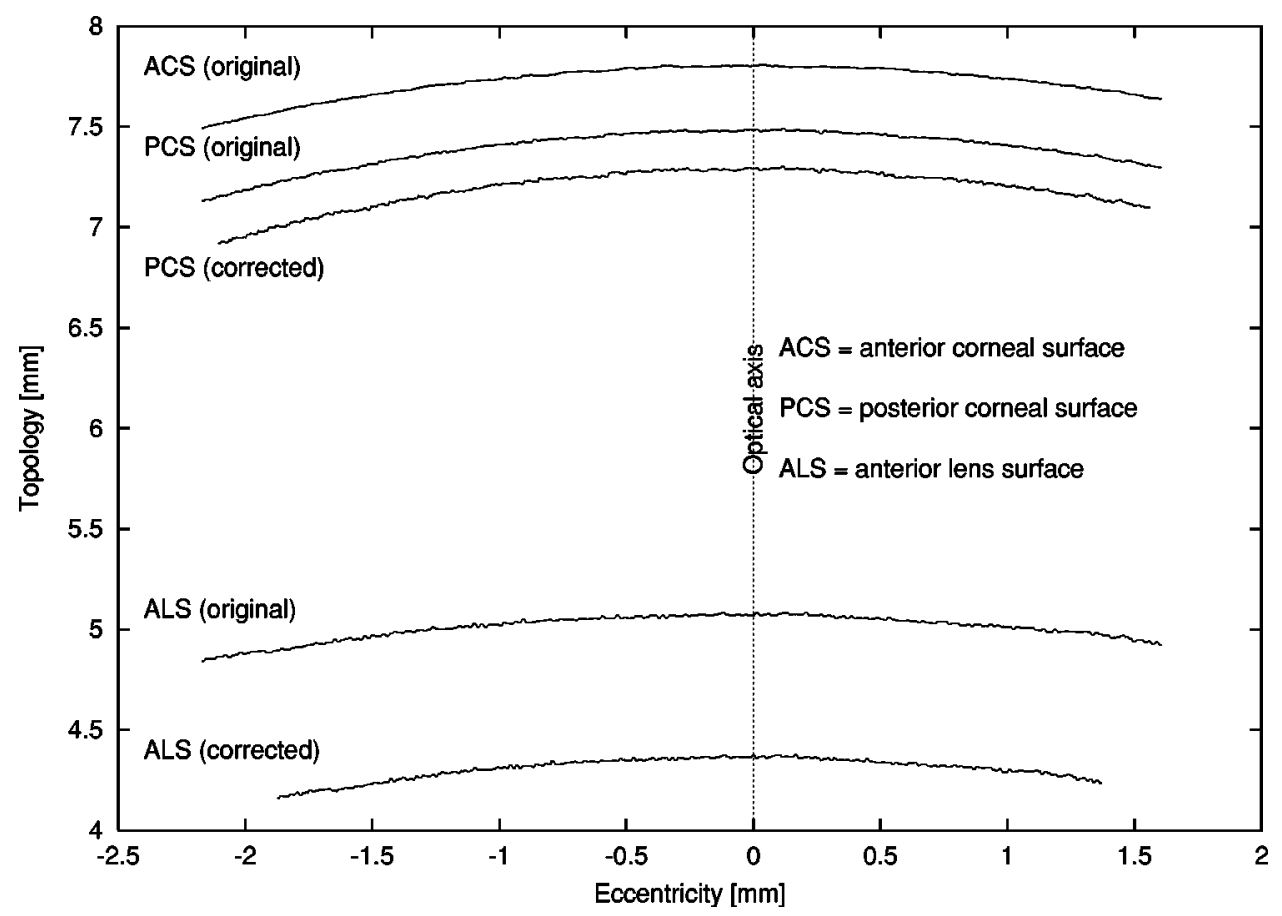

Fig. 5 Results of the refractive correction of a digital Scheimpflug photograph (Fig. 1).

PCS. The reason for this is that due to its nature, the circle fit routine $^{17}$ operates in two dimensions only. First, both Scheimpflug records must be corrected for the PCS only. Once the center of the sphere approximating the PCS has been determined, one can proceed with the refractive correction of the structure coordinates of the anterior lens surfaces in both Scheimpflug records, as described.

\section{Results}

As a numerical example, the described algorithm is applied to the data set used in Fink et al. ${ }^{2}$ The following results are obtained: Due to the optical/refractive correction by means of ray tracing the digital image taken by the Scheimpflug camera decreases in size perpendicular to the direction of the optical axis (see Fig. 5). As a consequence, the curvature radii of both the posterior corneal surface and the anterior lens surface are reduced as compared to the original Scheimpflug record (see Fig. 5 and Table 1). These results are consistent with Dubbelman et al. ${ }^{6}$ The corneal thickness and the anterior chamber

Table 1 Curvature radii for various refractive surfaces before and after refractive correction.

\begin{tabular}{lccc}
\hline & \multicolumn{3}{c}{ Curvature Radius } \\
\cline { 2 - 4 } & $\begin{array}{c}\text { Before } \\
\text { Correction } \\
(\mathrm{mm})\end{array}$ & $\begin{array}{c}\text { After } \\
\text { Correction } \\
(\mathrm{mm})\end{array}$ & $\begin{array}{c}\text { Change } \\
(\%)\end{array}$ \\
\hline Refractive Surface & 7.94 & - & - \\
Anterior corneal surface & 6.84 & 6.07 & -11.3 \\
Posterior corneal surface & 9.19 & 7.82 & -14.9 \\
Anterior lens surface & & &
\end{tabular}

depth are increased (see Fig. 5). Table 2 shows in particular the results for the central corneal thickness and the central anterior chamber depth.

\section{Discussion}

The main focus of this paper is the description of the theoretical underpinning of the refractive correction method. Thus, further testing of the algorithm in collaboration with clinicians and possible resulting modifications to the theory put forward in this paper are certainly necessary but beyond the scope of this paper. Further, the method presented here must be counterchecked by other measurement techniques such as A scan ultrasonography of the eye. However, both techniques, A scan ultrasonography and slitlamp Scheimpflug photography, have their respective drawbacks: ultrasonography requires the velocity of sound in the lens, which may be age-dependent, whereas Scheimpflug photography requires knowing the refractive index of the cornea, the anterior chamber, and the eye lens to enable correction of the photographs for the distortion due to the refraction of the cornea and lens (after Dubbelman et $\mathrm{al}^{8}{ }^{\circ}$.

One of the major advantages of the algorithm described in this paper is the fact that it is based on digital-CCD-recorded

Table 2 Biometrical data before and after refractive correction.

\begin{tabular}{lccc}
\hline Observable & $\begin{array}{c}\text { Before } \\
\text { Correction } \\
(\mathrm{mm})\end{array}$ & $\begin{array}{c}\text { After } \\
\text { Correction } \\
(\mathrm{mm})\end{array}$ & $\begin{array}{c}\text { Change } \\
(\%)\end{array}$ \\
\hline Central corneal thickness & 0.36 & 0.57 & +58.3 \\
Central anterior chamber depth & 1.84 & 2.32 & +26.1 \\
\hline
\end{tabular}


Scheimpflug photographs instead of digitally scanned Scheimpflug photographs (see Richards et al. ${ }^{4}$ and Kampfer et al. ${ }^{5}$ ). Thus, additional errors are avoided in the analysis of the original Scheimpflug photographs, namely inaccuracies that occur by processing and scanning of the original Scheimpflug photograph. Since photorefractive keratectomy (PRK) has become a fashionable method to correct for myopia and hyperopia, the technique presented in this paper enables a good estimate of the corneal thickness, which is crucial in the consideration of refractive surgery.

As the development of CCD chips rapidly moves on, CCD-chip-based cameras (digital cameras) with much higher optical resolution are becoming available, thus enabling an increasing refinement in the estimation of biometric quantities within the eye.

Access to the posterior eye lens surface in addition to the anterior eye lens surface via digital Scheimpflug photography may elucidate the accommodation process of the crystalline lens. The estimation of the curvature radii of both the anterior and posterior eye lens surfaces by means of the algorithm presented in this paper may enable monitoring the change of the eye lens shape as well as a possible dislocation of the eye lens. Therefore, questions may be answered concerning whether only the change in shape and location of the eye lens accounts for the necessary amount of accommodation, or whether some additional internal changes within the crystalline lens play a role, e.g., changes in refractive index (see also Dubbelman et al. ${ }^{9}$ ).

A more realistic extension of the correction algorithm could be achieved by using, e.g., 3-D B-splines (for a good introduction see Rogers and Adams ${ }^{20}$ and references given there) instead of spherical approximations for fitting both the anterior and the posterior corneal surfaces. This would enable us to investigate astigmatism and would produce even more accurate biometrical data from digital Scheimpflug photographs. However, for the application of 3-D B-splines, many Scheimpflug photographs of the same eye are required to cover the topology of the entire eye because 3-D B-splines operate on 3-D data sets and cannot be restricted to the 2-D Scheimpflug record plane under consideration.

\section{Acknowledgments}

I would like to thank H. J. Huebscher and T. Seiler for valuable discussions and advice. I am also grateful for reviewer A's annotation.

\section{References}

1. T. Scheimpflug, "Der Photoperspektograph und Seine Anwendung," Photogr. Korresp. 43, 516 (1906).
2. W. Fink, H. J. Huebscher, and T. Seiler, "Correction of biometric data derived from digital Scheimpflug records," Invest. Ophthalmol. Vis. Sci. 39(4), S1033, (1998) (ARVO abstract no. 4771).

3. H. J. Huebscher, W. Fink, D. Steinbrück, and T. Seiler, "Scheimpflug records without distortion-a mythos?" Ophthalmic Res. 31(2), 134139 (1998).

4. D. W. Richards, S. R. Russell, and D. R. Anderson, "A method for improved biometry of the anterior chamber with a Scheimpflug technique," Invest. Ophthalmol. Visual Sci. 29(12), 1826-1835 (1998).

5. T. Kampfer, A. Wegener, V. Dragomirescu, and O. Hockwin, "Improved biometry of the anterior eye segment," Ophthalmic Res. 21, 239-248 (1989).

6. M. Dubbelman, H. A. Weeber, G. L. van der Heijde, and H. J. VölkerDieben, "Radius and asphericity of the posterior corneal surface determined by corrected Scheimpflug photography," Acta Ophthalmol. Scand. 80(4), 379-383 (2002).

7. M. Dubbelman and G. L. van der Heijde, "The shape of the aging human lens: curvature equivalent refractive index and the lens paradoxon," Vision Res. 41(14), 1867-1877 (2001).

8. M. Dubbelman, G. L. van der Heijde, and H. A. Weeber, "The thickness of the aging human lens obtained from corrected Scheimpflug images," Optom. Vision Sci. 78(6), 411-416 (2001).

9. M. Dubbelman, G. L. van der Heijde, H. A. Weeber, and G. F. Vrensen, "Changes in the internal structure of the human crystalline lens with age and accommodation," Vision Res. 43(22), 2363-2375 (2003).

10. H. J. Huebscher and H. Schmidt, "Online Scheimpflug imaging and its potential for in-vivo examination of cornea and lens," Ophthalmic Res. 26(1), 33-38 (1994).

11. A. Hachicha, S. Simon, J. Samson, and K. Hanna, "The use of graylevel information and fitting techniques for precise measurement of corneal curvature and thickness," Comput. Vis. Graph. Image Process. 47(2), 131-164 (1989).

12. M. Born and E. Wolf, Principles of Optics, 5. ed., pp. 190-202, Pergamon Press, Oxford (1975).

13. W. Fink, A. Frohn, U. Schiefer, E. W. Schmid, and N. Wendelstein, "A ray tracer for ophthalmological applications," Ger. J. Ophthalmol. 5, 118-125 (1996).

14. W. Fink, A. Frohn, U. Schiefer, E. W. Schmid, N. Wendelstein, and E. Zrenner, "Visuelle Abbildung bei hohen Ametropien: Computergestützte Simulation mittels strahlen-optischer Rechnungen," Klin. Mbl. Augenheilk. 208, 472-476 (1996).

15. T. Kashiwagi and P. M. Khu, "Ray tracing error correction in ophthalmic optics," J. Cataract Refractive Surg. 17, 194-198 (1991).

16. W. Trendelenburg, M. Monjé, I. Schmidt, and E. Schütz, Der Gesichtssinn, 2nd ed., Springer, Berlin, Göttingen, Heidelberg (1961).

17. S. M. Thomas and Y. T. Chan, "A simple approach for the estimation of circular arc center and its radius," Comput. Vis. Graph. Image Process. 45, 362-370 (1989).

18. W. H. Press, B. P. Flannery, S. A. Teukolsky, and W. T. Vetterling, Numerical Recipes in C: The Art of Scientific Computing, pp. 286289, Cambridge University Press, Cambridge, NY (1991).

19. A. Gullstrand, Handbuch der Physiologischen Optik, 3rd ed., H. von Helmholtz, Vol. 1, p. 226, L. Voss, Hamburg, Leipzig (1909).

20. D. F. Rogers and J. A. Adams, Mathematical Elements for Computer Graphics, 2nd ed., McGraw-Hill, New York (1990). 